\title{
Association of lipoprotein(a) excess with early vein graft occlusions in middle-aged men undergoing coronary artery bypass surgery
}

\author{
Sergei N. Pokrovsky, PhD \\ Marat V. Ezhov, MD \\ Larisa N. Il'ina, MD \\ Olga I. Afanasieva, PhD \\ Valentin Y. Sinitsyn, MD \\ Andrey A. Shiriaev, MD \\ Renat S. Akchurin, MD
}

From the Cardiology Research Center, Moscow, Russia.

Received for publication May 31, 2002; revisions requested Sept 24, 2002; revisions received Oct 8, 2002; accepted for publication Oct 22, 2002.

Address for reprints: Sergei N. Pokrovsky, $\mathrm{PhD}$, Cardiology Research Center, 15a, 3rd Cherepkovskaya Street, Moscow 121552, Russia (E-mail: dr.pokrovsky@ cardio.ru).

J Thorac Cardiovasc Surg 2003;126:1071-5

Copyright $\odot 2003$ by The American Association for Thoracic Surgery

$0022-5223 / 2003 \$ 30.00+0$

doi:10.1016/S0022-5223(03)00365-9
Objective: To assess the relationship of lipoprotein(a) to early vein graft occlusions in patients after coronary artery bypass grafting.

Methods: We studied 102 male patients (mean age $52.3 \pm 8.6$ years) with chest pain occurrence during the first year (mean time $5.3 \pm 3.0$ months) after surgical myocardial revascularization. Graft patency was examined by electron-beam computed tomography $(\mathrm{n}=102)$ and quantitative coronary angiography $(\mathrm{n}=31)$.

Results: Patients were divided into 2 groups according to graft patency data: 66 (65\%) with occlusions and 36 (35\%) without occlusions at follow-up. No significant differences were found between the groups concerning age, smoking, family history of coronary heart disease, previous myocardial infarction, hypertension, serum lipids, and apolipoprotein B. Lipoprotein(a) level was significantly higher in patients with occluded grafts with a median (95\% confidence intervals) of $24 \mathrm{mg} / \mathrm{dL}$ (17-42 $\mathrm{mg} / \mathrm{dL}$ ) versus $12 \mathrm{mg} / \mathrm{dL}(6-24 \mathrm{mg} / \mathrm{dL})$ in patients with patent grafts, $P<.01$. More patients with nonoccluded grafts were taking statins postoperatively: $42 \%$ versus $18 \%$ of patients with occluded grafts, $P<.05$. The sensitivity and specificity of electron-beam computed tomography in revealing vein graft occlusion was close to $100 \%$.

Conclusion: There is an association between high lipoprotein(a) level and vein graft occlusions in middle-aged men during the first year after coronary artery bypass grafting. Use of statins is associated with a lower rate of vein graft occlusion. Electron-beam tomography could be useful for assessing graft occlusions.

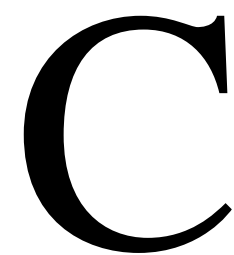
oronary artery bypass grafting $(\mathrm{CABG})$ is a key intervention in the treatment of severe symptomatic coronary heart disease (CHD). Serial angiographic studies reveal that $15 \%$ to $30 \%$ of vein grafts become stenosed within 1 year of surgery and nearly half of them are closed at 10 years. ${ }^{1}$ Early (within the first month after CABG) graft failure is generally caused by thrombosis, ${ }^{2}$ whereas intimal hyperplasia is the dominating process between 1 month and 1 year after implantation. $^{3}$ Beyond 1 year after operation, atherosclerosis of vein grafts can develop. ${ }^{3}$

Lipoprotein(a) (Lp[a]) is an emerging cardiovascular risk factor. Most prospective studies have demonstrated up to a twofold risk of coronary events in healthy subjects with high $\mathrm{Lp}(\mathrm{a})$ levels. ${ }^{4} \mathrm{Lp}(\mathrm{a})$ is a macromolecular complex of a lowdensity lipoprotein-like (LDL-like) particle and a unique glycoprotein, apolipoprotein(a) (apo[a]). The apo(a) molecule possesses high primary structure homology to 
TABLE 1. Baseline lipids of patients and healthy men

\begin{tabular}{lcc}
\hline Variable & Patients & Controls \\
\hline Lp(a) median (95\% Cl; mg/dL) & $16(13-24)$ & $8(4-13)^{*}$ \\
Lp(a) $\geq 30 \mathrm{mg} / \mathrm{dL}[\mathrm{n} \mathrm{( \% )]}$ & $39(38 \%)$ & $14(18.4 \%)^{*}$ \\
Total cholesterol (mmol/L) & $6.4 \pm 1.5$ & $5.7 \pm 1.0^{*}$ \\
HDL-C (mmol/L) & $1.0 \pm 0.5$ & $1.4 \pm 0.2^{*}$ \\
LDL-C (mmol/L) & $4.3 \pm 1.4$ & $3.7 \pm 0.9^{*}$ \\
TG (mmol/L) & $1.9 \pm 1.4$ & $1.9 \pm 0.9$ \\
\hline
\end{tabular}

HDL-C, High-density lipoprotein cholesterol; $L D L-C$, low-density lipoprotein cholesterol; $L p(a)$, lipoprotein(a); $T G$, triglycerides; $T C$, total cholesterol. ${ }^{*} P<.01$.

the plasminogen molecule. ${ }^{5}$ It was shown that $\mathrm{Lp}(\mathrm{a})$ can inhibit fibrinolysis ${ }^{6}$ and accumulates in atherosclerotic plaques of both native coronary arteries ${ }^{7}$ and vein grafts. ${ }^{8}$

Studies investigating the relationship between vein graft lesions and $\mathrm{Lp}(\mathrm{a})$ have reported conflicting results; some have seen a correlation with late-term patency ${ }^{9,10}$ but others failed to find a correlation with 1-year patency ${ }^{11}$ or 5 -year patency ${ }^{12}$ after operation. Given the risk of invasive angiography, ${ }^{12}$ noninvasive assessment of vein graft patency is desirable. Previously, it was shown that cardiac electronbeam computed tomography (EBCT) had close to $100 \%$ sensitivity and specificity in detection of vein graft patency. ${ }^{13}$ The aim of our study was to evaluate the association of $\mathrm{Lp}(\mathrm{a})$ as well as lipid levels and traditional risk factors with the first-year occlusions of vein grafts assessed by EBCT.

\section{Patients and Methods}

We studied 102 male patients (from 28 to 68 years old, mean 52.3 \pm 8.6 years) with chest pain appearance between the first and the twelfth month after myocardial revascularization by CABG (mean time after operation $5.3 \pm 3.0$ months). The study exclusion criteria were secondary dyslipidemia due to diabetes mellitus, dysfunction of thyroid gland, and liver or kidney failure.

The initial preoperative examination of all patients included medical history, routine clinical tests (electrocardiography [ECG], echocardiography), and coronary angiography. Before surgery, blood samples were collected from all patients and concentrations of total cholesterol, triglycerides, and high-density lipoprotein cholesterol (HDL-C) were tested immediately by specific enzymatic techniques of Boehringer Mannheim (Mannheim, Germany) on a Technicon RA-XT analyzer (GMI, Inc, Albertville, Minn). Concentration of apolipoprotein B (apoB) was measured by a turbodimetric method with monospecific polyclonal antibodies of a goat to human LDL. LDL cholesterol (LDL-C) was calculated by the Friedewald formula ${ }^{14}$ and, after adjustment for Lp(a)-cholesterol contribution, according to G. Dahlen. ${ }^{15} \mathrm{Lp}$ (a) concentration was determined by enzyme-linked immunosorbent assay (ELISA) using monospecific polyclonal sheep anti-human-apo(a) antibodies, as described previously. ${ }^{16}$ The intra-assay coefficient of variance has been determined to be $4.3 \%$ and $3.3 \%$ at 52 and 100 $\mathrm{mg} / \mathrm{dL}$, respectively, and the interassay coefficient of variance was $9.3 \%$ and $7.8 \%$, respectively. Cross-reactivity with LDL and plasminogen was negligible. Regression analysis obtained 95\% corre- lation in $\mathrm{Lp}$ (a) determination between our version of ELISA and a commercial kit (TintElisa Lp[a] Biopool International, Umea, Sweden).

For comparison of lipid parameters, we used a control group consisting of 76 healthy male donors without a personal history of cardiovascular disease.

Postoperatively, 100 (98\%) patients received aspirin and 27 $(26.5 \%)$ patients took hypolipidemic therapy by statins (simvastatin, $\mathrm{n}=17$; lovastatin, $\mathrm{n}=5$; fluvastatin, $\mathrm{n}=5$ ). At follow-up, all studied patients had chest pain; bicycle ergometry was performed in 100 of them. Although the exercise test results were positive in only 33 patients, all were subjected to graft examination, which was performed by EBCT with Imatron C-150 (GE Imatron, San Francisco, Calif). Each section was made during a 0.1 -second interval with a breath-hold and with ECG gating to minimize motion artifacts. The circulation time was determined with $120 \mathrm{~mL}$ of nonionic radiopaque preparation Omnipaque 350 (Amersham Corp, Arlington Heights, Ill) given by an automatic syringe into a cubital vein at a rate of 3.5 to $4.0 \mathrm{~mL} / \mathrm{s}$. The image processing and 3-dimensional reconstruction were carried out at a computer station MagicView 1000 (Siemens Healthcare Services, Oldbury, Bracknell, United Kingdom). The analysis of 244 vein and 92 arterial grafts was performed by 2 radiologists in a blinded manner. Also 31 patients were subjected to selective coronary and shunt angiography by the Judkins technique and a standard protocol used to define occlusions of vein graft proximal and distal anastomoses. Thus, both EBCT and coronary angiography were performed in 31 patients, with a total of 67 vein and 31 arterial grafts visualized. Occlusions of 54 vein and 3 arterial grafts were revealed by EBCT. These results were all confirmed by angiographic procedure. Thus, sensitivity and specificity of EBCT for graft occlusions in this sample was $100 \%$.

The study was conducted in accordance to the Declaration of Helsinki and approved by the local ethics committee. All patients gave written informed consent.

Statistical analysis was performed with the MedCalc program, version 5.00.020 (developed by Mathias Tschopp). In univariate analysis, continuous variables were compared by 2-tailed $t$ tests; the Mann-Whitney test was used for variables with non-Gaussian distribution. Discontinuous variables were compared by the $\chi^{2}$ and Fisher exact tests. Multivariate analysis was done by stepwise logistic regression to obtain predictive power of risk factors in the development of vein graft occlusions. Receiver operating characteristic (ROC) curve analysis was used to obtain threshold $\mathrm{Lp}$ (a) for graft occlusions. Data for discontinuous variables are presented as $\mathrm{n}(\%)$ and for continuous ones as mean \pm SD or as median $(95 \%$ confidence interval) for $\mathrm{Lp}(\mathrm{a})$.

\section{Results}

Of 102 patients studied, 47 (46\%) had arterial hypertension, $62(61 \%)$ had a family history of CHD, and $75(74 \%)$ were previous or current smokers. Elevated Lp(a) $(\geq 30 \mathrm{mg} / \mathrm{dL})$ was determined in 39 (38\%) patients. Lipid levels of patients and healthy controls are presented in Table 1. Preoperatively, all patients had severe angina pectoris with 2 $(11 \%)$ or $3(89 \%)$ diseased coronary vessels and $56(55 \%)$ 
of them already survived myocardial infarction. The mean number of distal anastomoses was $3.7 \pm 1.0$.

We performed bicycle ergometry in 100 patients. Of 33 patients with positive exercise test results, 32 had the symptoms of angina pectoris (classes I-IV according to the Canadian Cardiovascular Society) and 1 patient had silent ischemia. Atypical angina was present in 68 patients. Of 208 single vein grafts examined, $76(36.5 \%)$ were occluded; of 19 Y-mode grafts examined, 8 (42\%) were occluded; and of 17 sequential grafts examined, $8(47 \%)$ were occluded. Occlusion of 1 or more vein grafts was demonstrated in 66 of 102 patients in our study. Patients were then evaluated according to graft status: $66(65 \%)$ with occlusions and 36 (35\%) without occlusions. Of 66 patients with occluded grafts, half had positive exercise test results. The mean number of occluded grafts in this subgroup was higher than in the other half with negative test results: $2.1 \pm 1.1$ versus $1.4 \pm 0.7, \mathrm{t}=3.084,64$ d.f., $P=.003$. No significant differences were found between the groups with or without occlusions concerning age, smoking, family history of CHD, previous myocardial infarction, or hypertension (Table 2). More patients with nonoccluded grafts were taking statins postoperatively: 15 of $36(42 \%)$ versus 12 of 66 $(18 \%)$ with occluded grafts $(P<.05)$. Concentrations of serum total cholesterol, triglycerides, HDL-C, LDL-C, and apoB did not differ between the groups significantly, while Lp(a) level was higher in patients with occluded grafts.

Currently, the normal range of $\mathrm{Lp}$ (a) concentration is considered to be less than $30 \mathrm{mg} / \mathrm{dL}$. The number of patients with occluded grafts was significantly higher if the $\mathrm{Lp}(\mathrm{a})$ level was more than $30 \mathrm{mg} / \mathrm{dL}: 32$ of 39 (82\%) compared with 34 of $63(54 \%)$ if $\mathrm{Lp}(\mathrm{a})<30 \mathrm{mg} / \mathrm{dL}, P=.005$. ROC curve analysis revealed an $\mathrm{Lp}$ (a) threshold level of 28 $\mathrm{mg} / \mathrm{dL}$ (sensitivity $49 \%$, specificity $81 \%$, positive predictive value $82 \%$, negative predictive value for $\geq 1$ occluded graft $46 \%)$.

Arterial graft occlusions were detected less frequently than vein graft occlusions: only 5 of $92(5.4 \%)$ were occluded during the first year after operation. There were no differences in classic risk factors and lipid values between patients with patent and occluded arterial grafts.

Twenty-seven patients received hypolipidemic therapy with statins after operation. We compared them with 68 patients without any hypolipidemic treatment (7 patients had taken fibrates or niacin and were not considered in the analysis). Total cholesterol and LDL-C concentrations were significantly lower in the first group, whereas other lipid values did not differ (Table 3). There were more patients with patent vein grafts among those receiving statins: 15 (55\%) versus 21 (28\%), $P=.02$. Of 39 patients with elevated $L p(a), 10$ received statins after the operation. They had a significantly lower frequency of vein graft occlusion
TABLE 2. Comparison of patients with and without occluded vein grafts

\begin{tabular}{lcc}
\hline Variable & $\begin{array}{c}\text { Non-occluded } \\
\text { vein grafts }\end{array}$ & $\begin{array}{c}\text { Occluded vein } \\
\text { grafts }\end{array}$ \\
\hline Patients (n) & 36 & 66 \\
Age (y) & $51 \pm 9$ & $53 \pm 9$ \\
Time after CABG (mo) & $5.0 \pm 2.9$ & $5.8 \pm 3.0$ \\
Current smokers & $10(26 \%)$ & $20(30 \%)$ \\
Previous myocardial infarction & $18(46 \%)$ & $38(57 \%)$ \\
Family history of CHD & $21(54 \%)$ & $41(61 \%)$ \\
Hypertension & $16(41 \%)$ & $31(46 \%)$ \\
Treatment with statins after CABG & $15(42 \%)$ & $12(18 \%)^{*}$ \\
Lp(a) (mg/dL median [95\% Cl]) & $12[6-24]$ & $24[17-42] \dagger$ \\
Total cholesterol (mmol/L) & $6.2 \pm 1.3$ & $6.5 \pm 1.6$ \\
Triglycerides (mmol/L) & $1.9 \pm 1.8$ & $1.9 \pm 1.2$ \\
LDL-C (mmol/L) & $4.4 \pm 1.2$ & $4.6 \pm 1.5$ \\
LDL-Cc (mmol/L) & $4.3 \pm 1.2$ & $4.3 \pm 1.5$ \\
HDL-C (mmol/L) & $1.1 \pm 1.5$ & $1.0 \pm 0.4$ \\
Apolipoprotein B (mg/dL) & $138 \pm 56$ & $138 \pm 54$ \\
\hline
\end{tabular}

$\overline{L D L-C c, \text { LDL-cholesterol corrected for } \mathrm{Lp}(\mathrm{a}) \text {-cholesterol, as was proposed }}$ by G. Dahlen. ${ }^{14}$

${ }^{*} P<.05$.

$\dagger P<.01$.

than the remaining 29 patients: $5(50 \%)$ versus $26(90 \%), P$ $<.05$.

In univariate rank sum correlation analysis, $\mathrm{Lp}(\mathrm{a})(\mathrm{r}=$ $0.230, P=.02)$, statin therapy $(\mathrm{r}=0.197, P=.048)$, and HDL-C ( $\mathrm{r}=-0.193, P=.07)$ were related to vein graft occlusions. In a multivariate stepwise logistic regression model, the only predictors of vein graft occlusions were Lp(a) level $(P=.04)$ and statin therapy $(P=.02)$.

\section{Discussion}

Our study demonstrated the association between Lp(a) level and vein graft occlusions within the first year after CABG. Two other retrospective studies ${ }^{9,10}$ also revealed the relation of $L p(a)$ with vein graft lesions, but within several years after operation when there is a natural course of atherosclerosis of both native coronary arteries and grafts. In a prospective 5-year trial of 176 unselected patients with CABG, high $\mathrm{Lp}$ (a) concentration tended to be associated with obstructive changes in vein grafts, whereas mean levels of serum lipids and lipoproteins, other traditional risk factors for atherosclerosis, and subcutaneous fatty acid composition were similar in patients with and without graft occlusion. ${ }^{17}$ Other prospective trials did not reveal an association of $\mathrm{Lp}$ (a) level with clinical and angiographic outcome 5 years after CABG. ${ }^{12,18}$

Our main finding is the demonstration of the relationship of $L p(a)$ excess with vein graft occlusions in the first months after operation when the processes of thrombosis and intimal hyperplasia are prevailing. ${ }^{3}$ This could be explained by prothrombotic properties of the $\mathrm{Lp}$ (a) particle. ${ }^{6}$ In vitro studies have shown that $\mathrm{Lp}$ (a) stimulates smooth muscle 
TABLE 3. Lipid profile and treatment with statins after operation

\begin{tabular}{lccc}
\hline Variable & $\begin{array}{c}\text { Without statins } \\
\text { (n= 68) }\end{array}$ & $\begin{array}{c}\text { With statins } \\
\text { (n = 27) }\end{array}$ & \multicolumn{1}{c}{} \\
\hline Lp(a) (mg/dL median [95\% Cl]) & $23[15-33]$ & $18[10-36]$ & .5 \\
Total cholesterol (mmol/L baseline) & $6.6 \pm 1.5$ & $7.0 \pm 1.3$ & .2 \\
Total cholesterol (mmol/L at follow-up) & $6.6 \pm 1.5$ & $5.6 \pm 1.4 \dagger$ & $2.0 \pm 1.8$ \\
Triglycerides (mmol/L baseline) & $1.8 \pm 1.8$ & $1.4 \pm 1.1^{*}$ & .01 \\
Triglycerides (mmol/L at follow-up) & $2.1 \pm 1.6$ & $3.9 \pm 1.3$ & .02 \\
LDL-C (mmol/L) & $4.7 \pm 1.4$ & $3.5 \pm 1.5$ & .01 \\
LDL-Cc (mmol/L) & $4.5 \pm 1.6$ & $1.1 \pm 0.6$ & .006 \\
HDL-C (mmol/L) & $1.0 \pm 0.4$ & $131 \pm 46$ & .3 \\
Apolipoprotein B (mg/dL) & $140 \pm 55$ & .5
\end{tabular}

LDL-Cc, LDL-cholesterol corrected for Lp(a)-cholesterol, as was proposed by G. Dahlen. ${ }^{14}$

${ }^{*} P<.05 ; \dagger P<.01$, versus baseline levels of total cholesterol and triglycerides.

cell proliferation, may compete for fibrin binding, interacts with cell plasminogen receptor, and accumulates in thrombi. ${ }^{19,20}$ Therefore, $\mathrm{Lp}(\mathrm{a})$ may interfere with fibrinolysis and support a prothrombotic state. However, a prospective study of 610 patients undergoing CABG did not find an effect of $\mathrm{Lp}$ (a) level on graft patency at 1 year after CABG. ${ }^{11}$ Shunt angiography was performed in $95 \%$ of patients at a mean $12.1 \pm 1.5$ months after surgery. One or more occlusions of vein graft were revealed in almost half of the patients. The explanation of the conflict between this and our studies could lie in differences of patient cohorts; the first one was unselected and consisted of both men (87\%) and women, whereas our patients were only symptomatic middle-aged men. Thus, possible reasons for this disagreement could be the difference in age, sex, and the combination of risk factors in studied groups. The high rate of graft occlusion in our study is explained by the inclusion of patients with symptoms of typical or atypical angina, though two thirds of them did not have signs of ischemia on ECG. Probably, stress-echocardiography could help to obtain ischemic signs in this subgroup, but this method was not included in the study protocol.

Some studies examined the role of hemostatic factors in prognosis after CABG. In one of these studies, angiography performed 10 days postoperatively showed graft occlusion in $23 \%$ of 82 patients. Patients with graft occlusion had significantly $(P<.001)$ higher preoperative plasminogen activator inhibitor activity than patients without occlusion. ${ }^{21}$ These results suggest that increased plasminogen activator inhibitor activity might have a positive predictive value for early thrombosis in patients undergoing CABG. Preoperative and postoperative determinations (performed on the first, third, and sixth postoperative days) of hemostatic factors were performed in 100 men who had undergone elective CABG for stable angina pectoris. ${ }^{22}$ Occlusion of one or more vein grafts within 3 months of surgery occurred in 23 of them. The percentage increase in plasma plasminogen activator inhibitor-1 activity and fibrinogen levels within the first postoperative week were significantly $(P<$ .05 ) higher in patients who subsequently were found to have vein graft occlusion. ${ }^{22} \mathrm{We}$ propose that in patients with high $\mathrm{Lp}$ (a) level there is an increased risk of thrombotic graft occlusion due to concurrent inhibition of plasminogen by $\mathrm{Lp}$ (a) particles.

We have determined that therapy with statins is associated with a lower frequency of graft occlusion even in the first year after CABG. In the Post-CABG trial, the investigators showed that statin therapy reduced the progression of vein graft disease, the incidence of vein graft occlusion, and the need for further revascularization. ${ }^{23}$ This large study was started at 1 to 11 years after $\mathrm{CABG}$ and angiography was repeated an average of 4.3 years after baseline. The hypolipidemic therapy in our study was begun soon after operation and, though it was not randomized, the use of statins was associated with significantly better graft functioning in first months after operation. The reduction of blood thrombogenicity and endothelial dysfunction, ${ }^{24}$ as well as anti-inflammatory effect, ${ }^{25,26}$ may be mechanisms by which statins improve graft function and patency much earlier than the retarding of atherosclerosis through hypolipidemic activity.

We found EBCT to have high sensitivity for graft occlusion. Previously, it was shown that evaluation for hemodynamically relevant stenosis was possible in $84 \%$ of cases (36 of 43 patent grafts) and yielded a sensitivity of $100 \%$ (5 of 5 high-grade stenoses correctly detected) and a specificity of $97 \% .{ }^{13}$ We performed a substudy to compare EBCT and coronary angiography and obtained comparable accuracy in the revelation of graft occlusion. Therefore, we believe that EBCT should be used more widely to assess the condition of coronary grafts.

In conclusion, we found a significant association between elevated $\mathrm{Lp}(\mathrm{a})$ level and vein graft occlusion in middle-aged men in their first postoperative year. Patients not receiving statins and with $\operatorname{Lp}(\mathrm{a})$ excess had the highest rate of vein graft occlusion (90\%). It would be useful to 
measure $\mathrm{Lp}$ (a) concentration before surgery because patients with $\mathrm{Lp}(\mathrm{a})$ of $30 \mathrm{mg} / \mathrm{dL}$ or greater are at higher risk of subsequent vein graft lesions. Currently, there is no drug therapy available to lower $\mathrm{Lp}(\mathrm{a})$. Specific extracorporeal $\mathrm{Lp}$ (a) removal by $\mathrm{Lp}$ (a) apheresis, a procedure first designed and performed by our group, may retard progression of atherosclerosis in patients with selective $L p(a)$ elevation. $^{27,28}$ Taking into consideration the data that cardiovascular risk associated with high $\mathrm{Lp}(\mathrm{a})$ level is markedly enhanced when LDL-C is also elevated, ${ }^{29}$ soon after CABG all patients with both higher and normal $\mathrm{Lp}$ (a) levels in association with elevated LDL should receive aggressive hypolipidemic therapy, with the aim of avoiding fast progression of atherosclerosis in the bypasses. There is a need for future work in the investigation of the pathophysiologic role of $\mathrm{Lp}$ (a) in atherothrombosis and, furthermore, large prospective clinical trials could provide clarity as to the use of measuring $\mathrm{Lp}$ (a) level.

\section{References}

1. Fuster V, Vorchheimer DA. Prevention of atherosclerosis in coronary artery bypass grafts. $N$ Engl J Med. 1997;336:212-3.

2. Cox JL, Chiasson DA, Gotlieb AI. Stranger in a strange land: the pathogenesis of saphenous vein graft stenosis with emphasis on structural and functional differences between veins and arteries. Prog Cardiovasc Dis. 1991;34:45-68.

3. Motwani JG, Topol EJ. Aortocoronary saphenous vein graft disease. Circulation. 1998;97:916-31.

4. Danesh J, Collins R, Peto R. Lipoprotein(a) and coronary heart disease: meta-analysis of prospective studies. Circulation. 2000;102: 1082-5.

5. MacLean JW, Tomlinson JE, Kuang WJ, Eaton DL, Chen EY, Fless $\mathrm{GM}$, et al. cDNA Sequence of human apolipoprotein(a) is homologous to plasminogen. Nature. 1987;330:132-7.

6. Loscalzo J, Weunfeld M, Fless GM, Scanu AM. Lipoprotein(a), fibrin binding, and plasminogen activation. Arteriosclerosis. 1990;10:240-6.

7. Rath M, Niendorf A, Reblin T, Dietel M, Krebber HJ, Beisiegel U. Detection and quantification of lipoprotein(a) in arterial wall of 107 coronary bypass patients. Arteriosclerosis. 1989;9:579-92.

8. Cushing GL, Gaubatz JW, Nava ML, Burdick BJ, Bocan TMA, Guyton JB, et al. Quantitation and localization of apolipoproteins (a) and $\mathrm{B}$ in coronary artery bypass vein grafts resected at reoperation. Arteriosclerosis. 1989;9:593-603.

9. Hoff HF, Beck GL, Skibinski CI, Jurgens G, O'Neil J, Kramer J, Lytle B. Serum Lp(a) level as a predictor of vein graft stenosis after coronary artery bypass surgery in patients. Circulation. 1988;77:1238-44.

10. Solymoss BC, Marcil M, Wesolowsca E, Lesperance J, Pelletier LC, Campeau L. Risk factors of venous aortocoronary bypass graft disease noted at late symptom-directed angiographic study. Can J Cardiol. 1993;9:80-4.

11. Eritsland J, Arnesen H, Seljeflot I, Abdelnoor M, Gronseth K, Berg K, et al. Influence of serum lipoprotein(a) and homocyst(e)ine levels on graft patency after coronary artery bypass grafting. Am J Cardiol. 1994;74:1099-102.
12. Skinner JS, Farrer M, Albers CJ, Piper K, Neil HA, Adams PC. Serum $\mathrm{Lp}$ (a) lipoprotein concentration is not associated with clinical and angiographic outcome five years after coronary artery bypass graft surgery. Heart. 1997;78:131-5.

13. Achenbach S, Moshage W, Ropers D, Nossen J, Bachmann K. Noninvasive, three-dimensional visualization of coronary artery bypass grafts by electron beam tomography. Am J Cardiol. 1997;79:856-61.

14. Friedewald WT, Levy RI, Fredrickson DS. Estimation of the concentration of low-density lipoprotein cholesterol in plasma, without use of the preparative ultracentrifuge. Clin Chem. 1972;18:499-502.

15. Dahlen GH. Incidence of Lp(a) lipoprotein among populations. In: Scanu AM, editor. Lipoprotein(a). San Diego: Academic Press; 1990. p. 151-73.

16. Afanasieva OI, Adamova IY, Benevolenskaya GF, Pokrovsky SN. Immuno-enzyme assay for lipoprotein(a) measurement. Bull Exp Biol Med (Rus). 1995;4:398-401.

17. Korpilahti K, Engblom E, Syvanne M, Hamalainen H, Puukka P, Vanttinen E, et al. Angiographic changes in saphenous vein grafts and atherosclerosis risk factors. A 5-year study with serial measurements of serum lipids and lipoproteins. Scand Cardiovasc J. 1998;32:343-51.

18. Linden T, Taddei-Peters W, Wilhelmsen L, Herlitz J, Karlsson T, Ullstrom C, et al. Serum lipids, lipoprotein(a), and apo(a) isoforms in patients with established coronary artery disease and their relation to disease and prognosis after coronary bypass surgery. Atherosclerosis. 1998;137:175-86.

19. Hajjar KA, Nachman RL. The role of lipoprotein(a) in atherogenesis and thrombosis. Ann Rev Med. 1996;47:423-42.

20. Hobbs HH, White AL. Lipoprotein(a): intrigues and insights. Curr Opin Lipidol. 1999;10:225-36.

21. Rifon J, Paramo JA, Panizo C, Montes R, Rocha E. The increase of plasminogen activator inhibitor activity is associated with graft occlusion in patients undergoing aorto-coronary bypass surgery. $\mathrm{Br} J$ Haematol. 1997;99:262-7.

22. Moor E, Blomback M, Silveira A, Wiman B, Cederlund K, Bergstrand $\mathrm{L}$, et al. Haemostatic function in patients undergoing coronary artery bypass grafting: perioperative perturbations and relations to saphenous vein graft closure. Thromb Res. 2000;98:39-49.

23. Post Coronary Artery Bypass Graft Trial Investigators. The effect of aggressive lowering of low-density lipoprotein cholesterol levels and low-dose anticoagulation on obstructive changes in saphenous-vein coronary-artery bypass grafts. N Engl J Med. 1997;336:153-62.

24. Rauch U, Osende JI, Chesebro JH, Fuster V, Vorchheimer DA, Harris $\mathrm{K}$, et al. Statins and cardiovascular diseases: the multiple effects of lipid-lowering therapy by statins. Atherosclerosis. 2000;153:181-9.

25. Ferro D, Parrotto S, Basili S, Alessandri C, Violi F. Simvastatin inhibits the monocyte expression of proinflammatory cytokines in patients with hypercholesterolemia. J Am Coll Cardiol. 2000;36:42731.

26. Musial J, Undas A, Gajewski P, Jankowski M, Sydor W, Szczeklik A. Anti-inflammatory effects of simvastatin in subjects with hypercholesterolemia. Int J Cardiol. 2001;77:247-53.

27. Pokrovsky SN, Sussekov AV, Afanasieva OI, Adamova IY, Lyakishev AA, Kukharchuk VV. Extracorporeal immunoadsorbtion for the specific removal of lipoprotein (a) [Lp(a) apheresis]: preliminary clinical data. Chem Phys Lipids. 1994;67/68:323-30.

28. Ullrich H, Lackner KJ, Schmitz G. Lipoprotein(a) apheresis in severe coronary heart disease: an immunoadsorption method. Artif Organs. 1998;22:135-9.

29. Maher VMG, Brown BG. Lipoprotein(a) and coronary heart disease. Curr Opin Lipidol. 1995;6:229-35. 